\title{
Search for associated production of charginos and neutralinos in the trilepton final state using $2.3 \mathrm{fb}^{-1}$ of data
}

\section{DØ Collaboration}

V.M. Abazov ak, B. Abbott ${ }^{\text {bx }}$, M. Abolins ${ }^{\text {bn }}$, B.S. Acharya ${ }^{a d}$, M. Adams ${ }^{\text {az }}$, T. Adams ax, E. Aguilo ${ }^{f}$, M. Ahsan bh, G.D. Alexeev ${ }^{\text {ak }}$, G. Alkhazov ao, A. Alton bm,1, G. Alverson ${ }^{\text {bl }}$, G.A. Alves ${ }^{\text {b }}$, M. Anastasoaie aj, L.S. Ancu ${ }^{\text {aj }}$, T. Andeen ${ }^{\text {bb }}$, B. Andrieu ${ }^{\text {q }}$, M.S. Anzelc ${ }^{\text {bb }}$, M. Aoki ay , Y. Arnoud ${ }^{\text {n }}$, M. Arov ${ }^{\text {bi }}$, M. Arthaud ${ }^{\mathrm{r}}$, A. Askew ${ }^{\mathrm{ax}, 2}$, B. Åsman ap, A.C.S. Assis Jesus ${ }^{\mathrm{c}}$, O. Atramentov ${ }^{\mathrm{ax}}$, C. Avila $^{\mathrm{h}}$, J. BackusMayes ${ }^{\mathrm{ce}}$, F. Badaud $^{\mathrm{m}}$, L. Bagby ay, B. Baldin ay, D.V. Bandurin ${ }^{\text {bh }}$, P. Banerjee ${ }^{\text {ad }}$, S. Banerjee ${ }^{\text {ad }}$, E. Barberis ${ }^{\text {bl }}$, A.-F. Barfuss ${ }^{\circ}$, P. Bargassa ${ }^{\text {cc }}$, P. Baringer ${ }^{\text {bg }}$, J. Barreto ${ }^{\text {b }}$, J.F. Bartlett ${ }^{\text {ay }}$, U. Bassler ${ }^{r}$, D. Bauer ${ }^{\text {ar }}$, S. Beale ${ }^{\text {f }}$, A. Bean ${ }^{\text {bg }}$, M. Begalli ${ }^{\text {, }}$ M. Begel ${ }^{\text {bv }}$, C. Belanger-Champagne ${ }^{\text {ap }}$, L. Bellantoni ${ }^{\text {ay }}$, A. Bellavance ay, J.A. Benitez ${ }^{\text {bn }}$, S.B. Beri ${ }^{a b}$, G. Bernardi ${ }^{q}$, R. Bernhard ${ }^{x}$, I. Bertram ${ }^{\text {aq }}$, M. Besançon ${ }^{r}$, R. Beuselinck ${ }^{\text {ar }}$, V.A. Bezzubov ${ }^{\text {an }}$, P.C. Bhat ${ }^{\text {ay }}$, V. Bhatnagar ${ }^{a b}$, G. Blazey ${ }^{\text {ba }}$, F. Blekman ${ }^{\text {ar }}$, S. Blessing ${ }^{a x}$, K. Bloom $^{\text {bp }}$, A. Boehnlein ${ }^{\text {ay }}$, D. Boline $^{\text {bk }}$, T.A. Bolton ${ }^{\text {bh }}$, E.E. Boos am, G. Borissov ${ }^{\text {aq }}$, T. Bose ${ }^{\text {bz }}$, A. Brandt ${ }^{\text {ca }}$, R. Brock ${ }^{\text {bn }}$, G. Brooijmans ${ }^{\text {bs }}$, A. Bross ay ${ }^{\text {, D. Brown }}{ }^{\text {, }}$ X.B. Bu ${ }^{\text {g }}$, N.J. Buchanan ${ }^{\text {ax }}$, D. Buchholz ${ }^{\text {bb }}$, M. Buehler ${ }^{c d}$, V. Buescher ${ }^{\mathrm{w}}$, V. Bunichev ${ }^{\mathrm{am}}$, S. Burdin ${ }^{\mathrm{aq}, 3}$, T.H. Burnett ${ }^{\text {ce }}$, C.P. Buszello ${ }^{\text {ar }}$, P. Calfayan ${ }^{\mathrm{z}}$, B. Calpas ${ }^{\mathrm{o}}$, S. Calvet ${ }^{\mathrm{P}}$, J. Cammin ${ }^{\text {bt }}$, M.A. Carrasco-Lizarraga ${ }^{\text {ah }}$, E. Carrera ${ }^{\mathrm{ax}}$, W. Carvalho ${ }^{\mathrm{c}}$, B.C.K. Casey $^{\text {ay }}$, H. Castilla-Valdez ${ }^{\text {ah }}$, S. Chakrabarti bu, D. Chakraborty ${ }^{\text {ba }}$, K.M. Chan ${ }^{\text {bd }}$, A. Chandra ${ }^{\text {aw }}$, E. Cheu ${ }^{\text {at }}$, D.K. Cho ${ }^{\text {bk }}$, S. Choi ${ }^{\text {ag }}$, B. Choudhary ac, L. Christofek ${ }^{\text {bz }}$, T. Christoudias ${ }^{\text {ar }}$, S. Cihangir ay, D. Claes ${ }^{\text {bp }}$, J. Clutter ${ }^{\text {bg }}$, M. Cooke ay, W.E. Cooper ${ }^{\text {ay }}$, M. Corcoran ${ }^{\mathrm{cc}}$, F. Couderc ${ }^{\mathrm{r}}$, M.-C. Cousinou ${ }^{\mathrm{o}}$,

S. Crépé-Renaudin ${ }^{n}$, V. Cuplov ${ }^{\text {bh }}$, D. Cutts ${ }^{\text {bz }}$, M. Ćwiok ${ }^{\text {ae }}$, H. da Motta ${ }^{\text {b }}$, A. Das ${ }^{\text {at }}$, G. Davies ${ }^{\text {ar }}$, K. De $^{\text {ca }}$, S.J. de Jong ${ }^{\text {aj }}$, E. De La Cruz-Burelo ${ }^{\text {ah }}$, C. De Oliveira Martins ${ }^{c}$, K. DeVaughan ${ }^{b p}$, F. Déliot ${ }^{r}$, M. Demarteau ay, R. Demina ${ }^{\text {bt }}$, D. Denisovay, S.P. Denisov an, S. Desai ay, H.T. Diehl ay, M. Diesburg ay, A. Dominguez $^{\text {bp }}$, T. Dorland ${ }^{c e}$, A. Dubey ${ }^{a c}$, L.V. Dudko $^{a m}$, L. Duflot ${ }^{\mathrm{P}}$, S.R. Dugad ${ }^{\text {ad }}$, D. Duggan ${ }^{\mathrm{ax}}$, A. Duperrin ${ }^{0}$, S. Dutt ${ }^{\mathrm{ab}}$, J. Dyer ${ }^{\text {bn }}$, A. Dyshkant ${ }^{\text {ba }}$, M. Eads ${ }^{\text {bp }}$, D. Edmunds ${ }^{\text {bn }}$, J. Ellison ${ }^{\text {aw }}$, V.D. Elvira ${ }^{\text {ay }}$, Y. Enari ${ }^{\text {bz }}$, S. Eno bj, P. Ermolov am,10, M. Escalier ${ }^{\circ}$, H. Evans ${ }^{\text {bc }}$, A. Evdokimov bv , V.N. Evdokimov an, A.V. Ferapontov ${ }^{\text {bh }}$, T. Ferbel ${ }^{\text {bj, bt }}$, F. Fiedler ${ }^{\mathrm{y}}$, F. Filthaut ${ }^{\mathrm{aj}}$, W. Fisher ${ }^{\text {ay }}$, H.E. Fisk ${ }^{\text {ay }}$, M. Fortner ${ }^{\text {ba }}$, H. Fox $^{\text {aq }}$, S. Fu ${ }^{\text {ay }}$, S. Fuess ${ }^{\text {ay }}$, T. Gadfort ${ }^{\text {bs }}$, C.F. Galea ${ }^{\text {aj }}$, C. Garcia bt, A. Garcia-Bellido ${ }^{\text {bt }}$, V. Gavrilov ${ }^{\text {al }}$, P. Gay ${ }^{\mathrm{m}}$, W. Geist ${ }^{\mathrm{s}}$, W. Geng ${ }^{\mathrm{o}, \mathrm{bn}}$, C.E. Gerber ${ }^{\mathrm{az}}$, Y. Gershtein ${ }^{\mathrm{ax}, 2}$, D. Gillberg ${ }^{\mathrm{f}}$, G. Ginther ${ }^{\mathrm{bt}}$, B. Gómez ${ }^{\mathrm{h}}$, A. Goussiou ${ }^{c e}$, P.D. Grannis bu, H. Greenlee ${ }^{\text {ay }}$, Z.D. Greenwood ${ }^{\text {bi }}$, E.M. Gregores ${ }^{d}$, G. Grenier ${ }^{\text {t, u }}$, Ph. Gris ${ }^{\mathrm{m}}$, J.-F. Grivaz ${ }^{\text {p }, ~ A . ~ G r o h s j e a n ~}{ }^{z}$, S. Grünendahl ay, M.W. Grünewald ae, F. Guo bu, J. Guo bu, G. Gutierrez $^{\text {ay }}$, P. Gutierrez ${ }^{\text {bx }}$, A. Haas ${ }^{\text {bs }}$, N.J. Hadley bj, P. Haefner ${ }^{\text {}}$, S. Hagopian ${ }^{\text {ax }}$, J. Haley bq, I. Hall bn, R.E. Hall ${ }^{\text {av }}$, L. Han ${ }^{\text {g, K. Harder }}{ }^{\text {as }}$, A. Harel ${ }^{\text {bt }}$, J.M. Hauptman ${ }^{\text {bf }}$, J. Hays ${ }^{\text {ar }}$, T. Hebbeker ${ }^{\text {v }}$, D. Hedin ${ }^{\text {ba }}$, J.G. Hegeman ai , A.P. Heinson ${ }^{\text {aw }}$, U. Heintz ${ }^{\text {bk }}$, C. Hensel ${ }^{\text {w }}{ }^{4}$, K. Herner ${ }^{\text {bu }}$, G. Hesketh ${ }^{\text {bl }}$, M.D. Hildreth ${ }^{\text {bd }}$, R. Hirosky ${ }^{\text {cd }}$, T. Hoang ax, J.D. Hobbs bu, B. Hoeneisen ${ }^{\text {l, M. Hohlfeld }}{ }^{\text {w }}$, S. Hossain bx, P. Houben ai, Y. Hu bu, Z. Hubacek ${ }^{j}$, N. Huske ${ }^{q}$, V. Hynek $^{i}$, I. Iashvili ${ }^{\text {br }}$, R. Illingworth ${ }^{\text {ay }}$, A.S. Ito ${ }^{\text {ay }}$, S. Jabeen ${ }^{\text {bk }}$, M. Jaffrép,

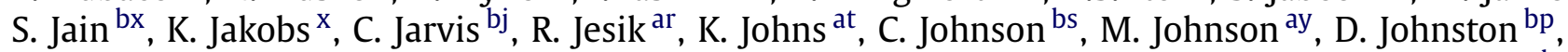
A. Jonckheere ${ }^{\text {ay }}$, P. Jonsson ${ }^{\text {ar }}$, A. Juste ${ }^{\text {ay }}$, E. Kajfasz ${ }^{\circ}$, D. Karmanov am, P.A. Kasper ay, I. Katsanos ${ }^{\text {bs }}$, V. Kaushik ${ }^{\text {ca }}$, R. Kehoe ${ }^{\text {cb }}$, S. Kermiche ${ }^{o}$, N. Khalatyan ay, A. Khanov by, A. Kharchilava br, Y.N. Kharzheev ${ }^{\text {ak }}$, D. Khatidze $^{\text {bs }}$, T.J. Kim af ${ }^{\text {, M.H. Kirby }}{ }^{\text {bb }}$, M. Kirsch ${ }^{\text {, }}$, B. Klima ay , J.M. Kohli ${ }^{\text {ab }}$, J.-P. Konrath ${ }^{\mathrm{x}}$, A.V. Kozelov an, J. Kraus bn, T. Kuhl ${ }^{y}$, A. Kumar br , A. Kupco ${ }^{k}$, T. Kurčat, u, V.A. Kuzmin am, J. Kvita ${ }^{\mathrm{i}}$, 


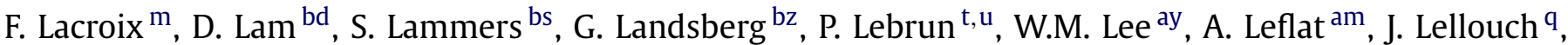
J. Li ${ }^{\text {ca, } 10}$, L. Li ${ }^{\text {aw }}$, Q.Z. Li ${ }^{\text {ay }}$, S.M. Lietti ${ }^{\text {e }}$, J.K. Lim ${ }^{\text {af }}$, J.G.R. Lima ${ }^{\text {ba }}$, D. Lincoln ${ }^{\text {ay }}$, J. Linnemann ${ }^{\text {bn }}$, V.V. Lipaev ${ }^{\text {an }}$, R. Lipton ${ }^{\text {ay }}$, Y. Liu ${ }^{g}$, Z. Liu ${ }^{f}$, A. Lobodenko ${ }^{\text {ao }}$, M. Lokajicek ${ }^{k}$, P. Love ${ }^{\text {aq }}$, H.J. Lubatti ${ }^{\text {ce }}$, R. Luna-Garcia ${ }^{\text {ah, }}{ }^{\text {, A.L. Lyon }}{ }^{\text {ay }}$, A.K.A. Maciel ${ }^{\text {b }}$, D. Mackin ${ }^{c c}$, R.J. Madaras ${ }^{\text {au }}$, P. Mättig aa ${ }^{\text {, }}$ A. Magerkurth ${ }^{\text {bm }}$, P.K. Mal ${ }^{\text {ce }}$, H.B. Malbouisson ${ }^{c}$, S. Malik bp, V.L. Malyshev ak, Y. Maravin ${ }^{\text {bh }}$, B. Martin ${ }^{\text {n }}$, R. McCarthy ${ }^{\text {bu }}$, M.M. Meijer aj, A. Melnitchouk ${ }^{\text {bo }}$, L. Mendoza ${ }^{\text {h }}$, P.G. Mercadante ${ }^{\mathrm{e}}$, M. Merkin am, K.W. Merritt ${ }^{\text {ay }}$, A. Meyer ${ }^{\mathrm{v}}$, J. Meyer ${ }^{\mathrm{w}, 4}$, J. Mitrevski ${ }^{\mathrm{bs}}$, R.K. Mommsen ${ }^{\mathrm{as}}$, N.K. Mondal ${ }^{\text {ad }}$, R.W. Moore ${ }^{\mathrm{f}}$, T. Moulik ${ }^{\text {bg }}$, G.S. Muanza ${ }^{\circ}$, M. Mulhearn ${ }^{\text {bs }}$, O. Mundal $^{\text {w }}$, L. Mundim ${ }^{\text {c }}$, E. Nagy ${ }^{\text {o }}$, M. Naimuddin ${ }^{\text {ay }}$, M. Narain ${ }^{\text {bz }}$, H.A. Neal ${ }^{\text {bm }}$, J.P. Negret ${ }^{h}$, P. Neustroev ${ }^{a o}$, H. Nilsen ${ }^{x}$, H. Nogima ${ }^{c}$, S.F. Novaes ${ }^{e}$, T. Nunnemann ${ }^{z}$, D.C. O’Neil ${ }^{\mathrm{f}}$, G. Obrant ${ }^{\text {ao }}$, C. Ochando $^{\mathrm{P}}$, D. Onoprienko ${ }^{\text {bh }}$, N. Oshima ay, N. Osman ar, J. Osta ${ }^{b d}$, R. Otec ${ }^{\mathrm{j}}$, G.J. Otero y Garzón ${ }^{\mathrm{a}}$, M. Owen ${ }^{\mathrm{as}}$, M. Padilla ${ }^{\mathrm{aw}}$, P. Padley ${ }^{\mathrm{cc}}$, M. Pangilinan ${ }^{\mathrm{bz}}$, N. Parashar be, S.-J. Parkw, ${ }^{\text {w }}$, S.K. Park ${ }^{\text {af }}$, J. Parsons ${ }^{\text {bs }}$, R. Partridge ${ }^{\text {bz }}$, N. Parua bc , A. Patwa bv,

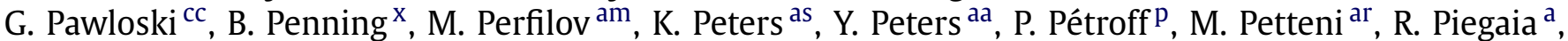
J. Piper $^{\text {bn }}$, M.-A. Pleier ${ }^{w}$, P.L.M. Podesta-Lerma ${ }^{\text {ah,6, }}$, V.M. Podstavkov ${ }^{\text {ay }}$, Y. Pogorelov ${ }^{\text {bd }}$, M.-E. Pol ${ }^{\text {b }}$, P. Polozov ${ }^{\text {al }}$, B.G. Pope ${ }^{\text {bn }}$, A.V. Popov ${ }^{\text {an }}$, C. Potter ${ }^{\text {f }}$, W.L. Prado da Silva ${ }^{\text {c }}$, H.B. Prosper ${ }^{\text {ax }}$, S. Protopopescu ${ }^{\text {bv }}$, J. Qian ${ }^{\text {bm }}$, A. Quadt ${ }^{\mathrm{w}}{ }^{4}$, B. Quinn ${ }^{\text {bo }}$, A. Rakitine ${ }^{\mathrm{aq}}$, M.S. Rangel ${ }^{\mathrm{b}}$, K. Ranjan ${ }^{\mathrm{ac}}$, P.N. Ratoff ${ }^{\text {aq }}$, P. Renkel ${ }^{\text {cb }}$, P. Rich ${ }^{\text {as }}$, M. Rijssenbeek ${ }^{\text {bu }}$, I. Ripp-Baudot ${ }^{\text {s }}$, F. Rizatdinova by ${ }^{\text {b. S. Robinson }}{ }^{\text {ar }}$, R.F. Rodrigues ${ }^{\text {c }}$, M. Rominsky ${ }^{\text {bx }}$, C. Royon ${ }^{r}$, P. Rubinov ${ }^{\text {ay }}$, R. Ruchti ${ }^{\text {bd }}$, G. Safronov ${ }^{\text {al }}$, G. Sajot ${ }^{\mathrm{n}}$,

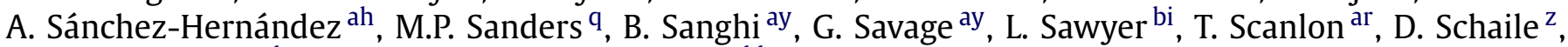
R.D. Schamberger ${ }^{\text {bu }}$, Y. Scheglov ao, H. Schellman ${ }^{\text {bb }}$, T. Schliephake aa ${ }^{\text {a S. Schlobohm }}{ }^{\text {ce }}$,

C. Schwanenberger as, R. Schwienhorst ${ }^{\text {bn }}$, J. Sekaric ax, H. Severini ${ }^{\text {bx }}$, E. Shabalina az, M. Shamim ${ }^{\text {bh }}$,

V. Shary ${ }^{r}$, A.A. Shchukin ${ }^{\text {an }}$, R.K. Shivpuri ${ }^{\text {ac }}$, V. Siccardi ${ }^{\text {s }}$, V. Simak ${ }^{j}$, V. Sirotenko ${ }^{\text {ay }}$, P. Skubic ${ }^{\text {bx }}$,

P. Slattery bt, D. Smirnov bd, G.R. Snow ${ }^{\text {bp }}{ }^{\text {, J. Snow }}{ }^{\text {bw }}$, S. Snyder ${ }^{\text {bv }}$, S. Söldner-Rembold ${ }^{\text {as }}$,

L. Sonnenschein q, A. Sopczak aq, M. Sosebee ${ }^{\text {ca }}$, K. Soustruznik ${ }^{\mathrm{i}}$, B. Spurlock ${ }^{\text {ca }}$, J. Stark ${ }^{\mathrm{n}}$, V. Stolin ${ }^{\text {al }}$, D.A. Stoyanova an, J. Strandberg ${ }^{\text {bm }}$, S. Strandberg ap, M.A. Strang ${ }^{\text {br }}$, E. Strauss ${ }^{\text {bu }}$, M. Strauss ${ }^{\text {bx }}$,

R. Ströhmer ${ }^{\text {, }}$ D. Strom ${ }^{\text {bb }}$, L. Stutte ${ }^{\text {ay }}$, S. Sumowidagdo ax, P. Svoisky aj, A. Sznajder ${ }^{c}$, A. Tanasijczuk ${ }^{\text {a }}$, W. Taylor ${ }^{\mathrm{f}}$, B. Tiller $^{\mathrm{z}}$, F. Tissandier ${ }^{\mathrm{m}}$, M. Titov ${ }^{\mathrm{r}}$, V.V. Tokmenin ${ }^{\text {ak }}$, I. Torchiani ${ }^{\mathrm{x}}$, D. Tsybychev ${ }^{\text {bu }}$, B. Tuchming ${ }^{r}$, C. Tully ${ }^{\text {bq }}$, P.M. Tuts ${ }^{\text {bs }}$, R. Unalan ${ }^{\text {bn }}$, L. Uvarov ${ }^{\text {ao }}$, S. Uvarov ${ }^{\text {ao }}$, S. Uzunyan ${ }^{\text {ba }}$, B. Vachon ${ }^{\mathrm{f}}$, P.J. van den Berg ${ }^{\mathrm{ai}}$, R. Van Kooten ${ }^{\mathrm{bc}}$, W.M. van Leeuwen ${ }^{\mathrm{ai}}$, N. Varelas ${ }^{\mathrm{az}}$, E.W. Varnes ${ }^{\text {at }}$, I.A. Vasilyev ${ }^{\text {an }}$, P. Verdier ${ }^{\text {t, }}$, L.S. Vertogradov ${ }^{\text {ak }}$, M. Verzocchi ${ }^{\text {ay }}$, D. Vilanova ${ }^{\mathrm{r}}$, F. Villeneuve-Seguier ${ }^{\text {ar }}$, P. Vint $^{\text {ar }}$, P. Vokac ${ }^{j}$, M. Voutilainen ${ }^{\text {bp, }}$, R. Wagner ${ }^{\text {bq }}$, H.D. Wahl ${ }^{\text {ax }}$, M.H.L.S. Wang ${ }^{\text {ay }}$, J. Warchol ${ }^{\text {bd }}$, G. Watts ${ }^{\text {ce }}$, M. Wayne ${ }^{\text {bd }}$, G. Weber ${ }^{\mathrm{y}}$, M. Weber ${ }^{\text {ay }, 8}$, L. Welty-Rieger ${ }^{\text {bc }}$, A. Wenger ${ }^{\mathrm{x}, 9}$, N. Wermes ${ }^{\mathrm{w}}$, M. Wetstein ${ }^{\text {bj }}$, A. White ${ }^{\text {ca }}$, D. Wicke aa , M.R.J. Williams ${ }^{\text {aq }}$, G.W. Wilson ${ }^{\text {bg }}$, S.J. Wimpenny ${ }^{\text {aw }}$, M. Wobisch ${ }^{\text {bi }}$, D.R. Wood ${ }^{\text {bl }}$, T.R. Wyatt ${ }^{\text {as }}$, Y. Xie ${ }^{\text {bz }}$, C. Xu ${ }^{\text {bm }}$, S. Yacoob ${ }^{\text {bb }}$, R. Yamada ay, W.-C. Yang as, T. Yasuda ay, Y.A. Yatsunenko ${ }^{\text {ak }}$, Z. Ye ${ }^{\text {ay }}$, H. Yin ${ }^{\mathrm{g}}$, K. Yip ${ }^{\text {bv }}$, H.D. Yoo ${ }^{\text {bz }}$, S.W. Youn ${ }^{\text {bb }}$, J. Yu ${ }^{\text {ca }}$, C. Zeitnitz $^{\text {aa }}$, S. Zelitch ${ }^{\text {cd }}$, T. Zhao ${ }^{\text {ce }}$, B. Zhou ${ }^{\text {bm }}$, J. Zhu ${ }^{\text {bu }}$, M. Zielinski ${ }^{\text {bt }}$, D. Zieminska ${ }^{\text {bc }}$, L. Zivkovic ${ }^{\text {bs }}$, V. Zutshi ${ }^{\text {ba }}$, E.G. Zverev ${ }^{\text {am }}$

\footnotetext{
a Universidad de Buenos Aires, Buenos Aires, Argentina

${ }^{\mathrm{b}}$ LAFEX, Centro Brasileiro de Pesquisas Físicas, Rio de Janeiro, Brazil

c Universidade do Estado do Rio de Janeiro, Rio de Janeiro, Brazil

d Universidade Federal do ABC, Santo André, Brazil

e Instituto de Física Teórica, Universidade Estadual Paulista, São Paulo, Brazil

${ }^{g}$ University of Science and Technology of China, Hefei, People's Republic of China

h Universidad de los Andes, Bogotá, Colombia

i Center for Particle Physics, Charles University, Prague, Czech Republic

j Czech Technical University, Prague, Czech Republic

${ }^{1}$ Universidad San Francisco de Quito, Quito, Ecuador

m LPC, Université Blaise Pascal, CNRS/IN2P3, Clermont, France

${ }^{\circ}$ CPPM, Aix-Marseille Université, CNRS/IN2P3, Marseille, France

p LAL, Université Paris-Sud, IN2P3/CNRS, Orsay, France

${ }^{q}$ LPNHE, IN2P3/CNRS, Universités Paris VI and VII, Paris, France

${ }^{\mathrm{r}}$ CEA, Irfu, SPP, Saclay, France

${ }^{s}$ IPHC, Université Louis Pasteur, CNRS/IN2P3, Strasbourg, France

${ }^{\mathrm{t}}$ IPNL, Université Lyon 1, CNRS/IN2P3, Villeurbanne, France

u Université de Lyon, Lyon, France

${ }^{v}$ III. Physikalisches Institut A, RWTH Aachen University, Aachen, Germany

${ }^{\mathrm{w}}$ Physikalisches Institut, Universität Bonn, Bonn, Germany

${ }^{x}$ Physikalisches Institut, Universität Freiburg, Freiburg, Germany
}

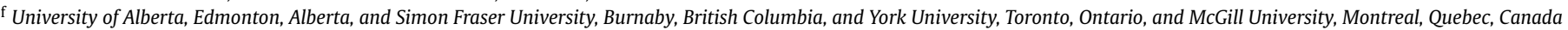

${ }^{\mathrm{k}}$ Center for Particle Physics, Institute of Physics, Academy of Sciences of the Czech Republic, Prague, Czech Republic

n LPSC, Université Joseph Fourier Grenoble 1, CNRS/IN2P3, Institut National Polytechnique de Grenoble, Grenoble, France 
y Institut für Physik, Universität Mainz, Mainz, Germany

${ }^{\mathrm{z}}$ Ludwig-Maximilians-Universität München, München, Germany

aa Fachbereich Physik, University of Wuppertal, Wuppertal, Germany

ab Panjab University, Chandigarh, India

ac Delhi University, Delhi, India

${ }^{\text {ad }}$ Tata Institute of Fundamental Research, Mumbai, India

ae University College Dublin, Dublin, Ireland

${ }^{\text {af }}$ Korea Detector Laboratory, Korea University, Seoul, Republic of Korea

ag SungKyunKwan University, Suwon, Republic of Korea

ah CINVESTAV, Mexico City, Mexico

ai FOM-Institute NIKHEF and University of Amsterdam/NIKHEF, Amsterdam, The Netherlands

aj Radboud University Nijmegen/NIKHEF, Nijmegen, The Netherlands

ak Joint Institute for Nuclear Research, Dubna, Russia

al Institute for Theoretical and Experimental Physics, Moscow, Russia

am Moscow State University, Moscow, Russia

an Institute for High Energy Physics, Protvino, Russia

ao Petersburg Nuclear Physics Institute, St. Petersburg, Russia

ap Lund University, Lund, and Royal Institute of Technology and Stockholm University, Stockholm, and Uppsala University, Uppsala, Sweden

aq Lancaster University, Lancaster, United Kingdom

ar Imperial College, London, United Kingdom

as University of Manchester, Manchester, United Kingdom

at University of Arizona, Tucson, AZ 85721, USA

au Lawrence Berkeley National Laboratory and University of California, Berkeley, CA 94720, USA

av California State University, Fresno, CA 93740, USA

aw University of California, Riverside, CA 92521, USA

ax Florida State University, Tallahassee, FL 32306, USA

ay Fermi National Accelerator Laboratory, Batavia, IL 60510, USA

az University of Illinois at Chicago, Chicago, IL 60607, USA

ba Northern Illinois University, DeKalb, IL 60115, USA

bb Northwestern University, Evanston, IL 60208, USA

bc Indiana University, Bloomington, IN 47405, USA

bd University of Notre Dame, Notre Dame, IN 46556, USA

be Purdue University Calumet, Hammond, IN 46323, USA

bf Iowa State University, Ames, IA 50011, USA

bg University of Kansas, Lawrence, KS 66045, USA

bh Kansas State University, Manhattan, KS 66506, USA

bi Louisiana Tech University, Ruston, LA 71272, USA

bj University of Maryland, College Park, MD 20742, USA

bk Boston University, Boston, MA 02215, USA

bl Northeastern University, Boston, MA 02115, USA

bm University of Michigan, Ann Arbor, MI 48109, USA

bn Michigan State University, East Lansing, MI 48824, USA

bo University of Mississippi, University, MS 38677, USA

bp University of Nebraska, Lincoln, NE 68588, USA

bq Princeton University, Princeton, NJ 08544, USA

br State University of New York, Buffalo, NY 14260, USA

bs Columbia University, New York, NY 10027, USA

bt University of Rochester, Rochester, NY 14627, USA

bu State University of New York, Stony Brook, NY 11794, USA

bv Brookhaven National Laboratory, Upton, NY 11973, USA

bw Langston University, Langston, OK 73050, USA

bx University of Oklahoma, Norman, OK 73019, USA

by Oklahoma State University, Stillwater, OK 74078, USA

bz Brown University, Providence, RI 02912, USA

ca University of Texas, Arlington, TX 76019, USA

cb Southern Methodist University, Dallas, TX 75275, USA

${ }^{c c}$ Rice University, Houston, TX 77005, USA

cd University of Virginia, Charlottesville, VA 22901, USA

ce University of Washington, Seattle, WA 98195, USA

\section{A R T I C L E I N F O}

\section{Article history:}

Received 8 January 2009

Received in revised form 30 July 2009

Accepted 7 August 2009

Available online 13 August 2009

Editor: L. Rolandi

\section{PACS:}

14.80.Ly

13.85.Rm

12.60.Jv

\section{A B S T R A C T}

We report the results of a search for associated production of charginos and neutralinos using a data set corresponding to an integrated luminosity of $2.3 \mathrm{fb}^{-1}$ collected with the Dø experiment during Run II of the Tevatron proton-antiproton collider. Final states containing three charged leptons and missing transverse energy are probed for a signal from supersymmetry with four dedicated trilepton event selections. No evidence for a signal is observed, and we set limits on the product of production cross section and leptonic branching fraction. Within minimal supergravity, these limits translate into bounds on $m_{0}$ and $m_{1 / 2}$ that are well beyond existing limits.

(c) 2009 Elsevier B.V. All rights reserved. 
Supersymmetry (SUSY) [1] is one of the most popular extensions of the standard model (SM). SUSY can solve the hierarchy problem, allows the unification of gauge couplings, and provides a dark matter candidate. The analyses presented in this Letter are based on the supersymmetric extension of the SM with minimal field content, the so-called minimal supersymmetric standard model (MSSM), which requires the addition of a SUSY partner for each SM particle, differing by half a unit in spin. The supersymmetric partners of charged and neutral Higgs and gauge bosons form two chargino $\left(\tilde{\chi}^{ \pm}\right)$and four neutralino $\left(\tilde{\chi}^{0}\right)$ mass eigenstates. Experiments at the CERN $e^{+} e^{-}$Collider (LEP) have set lower limits on the masses of SUSY particles. In particular, charginos with mass lower than $103.5 \mathrm{GeV}$ and sleptons $(\tilde{\ell})$ with mass below $95 \mathrm{GeV}$ are excluded [2]. The results presented here are the extensions of an earlier search for charginos and neutralinos by the Dø Collaboration based on $0.3 \mathrm{fb}^{-1}$ of data [3]. The CDF Collaboration has published limits for charginos and neutralinos using $2.0 \mathrm{fb}^{-1}$ of data [4].

In $p \bar{p}$ collisions, charginos and neutralinos can be produced in pairs via an off-shell $W$ boson or the exchange of squarks. They decay into fermions and the lightest neutralino $\tilde{\chi}_{1}^{0}$, which is assumed to be the lightest supersymmetric particle (LSP) and to escape undetected. This Letter describes the search for $p \bar{p} \rightarrow \tilde{\chi}_{1}^{ \pm} \tilde{\chi}_{2}^{0}$ in purely leptonic decay modes in final states with missing transverse energy $\mathbb{E}_{T}$ and three charged leptons $(e, \mu$ or $\tau$ ). This signature of three leptons can be particularly challenging in regions of parameter space where lepton momenta are very soft due to small mass differences of the SUSY particles. The analyses are based on $p \bar{p}$ collision data at a center-of-mass energy of $1.96 \mathrm{TeV}$ recorded with the DØ detector at the Fermilab Tevatron Collider between March 2002 and June 2007 corresponding to an integrated luminosity of $2.3 \mathrm{fb}^{-1}$, with the exception of the analysis using identified hadronic $\tau$ lepton decays, which is based on $1 \mathrm{fb}^{-1}$ of data.

The $\mathrm{D} \emptyset$ detector [5] consists of a central tracking system surrounded by a liquid-argon sampling calorimeter and a muon system. The inner tracking systems, a silicon microstrip tracker and a central fiber tracker, reside in an axial magnetic field of $2 \mathrm{~T}$. The $\eta$ coverage of the calorimeter extends down to pseudorapidities of $|\eta| \approx 4$, where $\eta=-\ln [\tan (\theta / 2)]$ and $\theta$ is the polar angle with respect to the proton beam direction. Muons are identified in the inner tracking system as well as in the outer muon system, which consists of three layers of tracking detectors and scintillator counters. An iron toroidal magnet providing a field of $1.8 \mathrm{~T}$ is located between the two innermost layers. The muon system provides coverage for muon identification up to $|\eta| \approx 2$. A threestage real-time trigger system reduces the total rate from $2.5 \mathrm{MHz}$ to about $100 \mathrm{~Hz}$. Events for the offline analyses are collected by a combination of single lepton, di-lepton, and lepton plus track triggers. Electrons and muons are selected by their specific energy deposition in the calorimeter and hits in the muon chambers, respectively. In addition, high momentum tracks matched to the

\footnotetext{
E-mail address: hohlfeld@fnal.gov (M. Hohlfeld).

1 Visitor from Augustana College, Sioux Falls, SD, USA.

2 Visitor from Rutgers University, Piscataway, NJ, USA.

3 Visitor from The University of Liverpool, Liverpool, UK.

4 Visitor from II. Physikalisches Institut, Georg-August-University, Göttingen, Germany.

5 Visitor from Centro de Investigacion en Computacion - IPN, Mexico City, Mexico.

6 Visitor from ECFM, Universidad Autonoma de Sinaloa, Culiacán, Mexico.

7 Visitor from Helsinki Institute of Physics, Helsinki, Finland.

8 Visitor from Universität Bern, Bern, Switzerland.

9 Visitor from Universität Zürich, Zürich, Switzerland.

10 Deceased.
}

objects in the calorimeter and muon system help to reduce the trigger rates.

Standard model and SUSY processes are simulated with the event generators PYTHIA [6] (Drell-Yan, di-boson, $\Upsilon$, and $t \bar{t}$ events) and ALPGEN [7] ( $W+$ jet $/ \gamma$ events). The simulation of the detector geometry and response is based on GEANT [8]. Detector noise and additional interactions are included using randomly triggered events recorded throughout the duration of the data-taking period. The predictions for the SM backgrounds are normalized using the next-to-leading (NLO) and, for Drell-Yan production, next-to-NLO theoretical cross sections, calculated using CTEQ6.1M parton distribution functions [9].

The contributions from multijet background are estimated using DØ data. For each analysis, samples dominated by multijet background are defined that are identical to the search samples except for reversed lepton identification requirements. In case of the electrons, jet-like electrons are selected based on the likelihood criterion (see below) while for the muons the isolation criteria (see below) are inverted. The normalization of these samples is performed at an early stage of the selection in a region of phase space that is dominated by multijet production.

The optimization of the analysis is done using minimal supergravity (mSUGRA) [10] as a reference model, in regions of parameter space with chargino, neutralino, and slepton masses ranging from 100 to $200 \mathrm{GeV}$. The mSUGRA scenario can be described by five independent parameters: the unified scalar and gaugino masses $m_{0}$ and $m_{1 / 2}$, the ratio of the vacuum expectation values of the two Higgs doublets, $\tan \beta$, the unified trilinear coupling $A_{0}$, and the sign of the Higgs mass parameter $\mu$. The SUSY spectra are calculated using sofTSUSY [11]. The selection criteria are optimized to achieve the best average expected limit under the assumption that no signal is present in the data. A modified frequentist approach [12] is used to calculate limits at the 95\% C.L. for each different final state and selection. Two choices of mSUGRA parameters $\left(m_{0}=150 \mathrm{GeV}\right.$ and $m_{1 / 2}=250$ (170) $\mathrm{GeV}$, with $\tan \beta=3$, $A_{0}=0$ and $\mu>0$ ) are used as a reference for a high- $p_{T}$ (low- $p_{T}$ ) signal, labeled SUSY1 (SUSY2) in the plots shown in the following.

The reconstruction of isolated electrons exploits their characteristic energy deposition in the calorimeter. All electromagnetic clusters with $|\eta|<3.2$ are considered. A track is required to point to the calorimeter energy cluster, and the track momentum and the calorimeter energy must be consistent. A likelihood discriminant is used to reject background contributions from jets, based on their differences in transverse and longitudinal shower shape as well as differences in isolation in the tracker. The selection of muons relies on a combination of tracks in the central tracker and pattern of hits in the muon detector within $|\eta|<2.0$. Isolation criteria are imposed in both the tracker and the calorimeter in order to suppress background contributions from jets. Two different type of muons, referred to as "loose" and "tight", are used in the analyses. The classification of loose and tight muons depends on the level of calorimeter and tracker isolation of the candidate. The isolation in the calorimeter is based on the cell energies in a hollow cone of $0.1<\Delta \mathcal{R}<0.4$, where $\Delta \mathcal{R}=\sqrt{(\Delta \eta)^{2}+(\Delta \phi)^{2}}$. The tracker isolation is defined as the scalar sum of the transverse momenta of all tracks in a cone of $\Delta \mathcal{R}<0.4$ around the muon track. The energies for both calorimeter and tracker isolation are required to be less than $4 \mathrm{GeV}(2.5 \mathrm{GeV})$ for loose (tight) muons. Reconstruction efficiencies for both $e$ and $\mu$ are measured using $Z \rightarrow \ell \ell$ events, and the efficiencies in the Monte Carlo (MC) simulation are corrected for known differences according to the measurements in the data.

The reconstruction of hadronically decaying $\tau$ leptons is seeded by calorimeter clusters or tracks [13] with $|\eta|<2.5$. According to their signature in the detector, they are classified into three types. 
Table 1

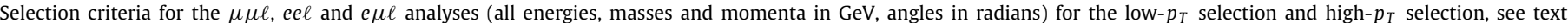
for further details.

\begin{tabular}{|c|c|c|c|c|c|c|c|}
\hline & \multirow[t]{2}{*}{ Selection } & \multicolumn{2}{|l|}{$\mu \mu \ell$} & \multicolumn{2}{|l|}{ ee $\ell$} & \multicolumn{2}{|l|}{$e \mu \ell$} \\
\hline & & low $p_{T}$ & high $p_{T}$ & low $p_{T}$ & high $p_{T}$ & low $p_{T}$ & high $p_{T}$ \\
\hline I & $p_{T}^{\ell 1}, p_{T}^{\ell 2}$ & $>12,>8$ & $>18,>16$ & $>12,>8$ & $>20,>10$ & $>12,>8^{a}$ & $>15,>15$ \\
\hline II & $\begin{array}{l}m_{\ell_{1} \ell_{2}}{ }^{\mathrm{b}} \\
\Delta \phi_{\ell_{1} \ell_{2}}\end{array}$ & $\begin{array}{l}\in[20,60] \\
<2.9\end{array}$ & $\begin{array}{l}\in[0,75] \\
<2.9\end{array}$ & $\begin{array}{l}\in[18,60] \\
<2.9\end{array}$ & $\begin{array}{l}\in[0,75] \\
<2.9\end{array}$ & $\begin{array}{l}- \\
-\end{array}$ & $\begin{array}{l}- \\
-\end{array}$ \\
\hline III & $\begin{array}{l}\not_{T} \\
\operatorname{Sig}\left(\not k_{T}\right) \\
m_{T}^{\min } \\
\text { jet-veto } H_{T}\end{array}$ & $\begin{array}{l}>20 \\
>8 \\
>20 \\
-\end{array}$ & $\begin{array}{l}>20 \\
>8 \\
>20 \\
<80\end{array}$ & $\begin{array}{l}>22 \\
>8 \\
>20 \\
-\end{array}$ & $\begin{array}{l}>20 \\
>8 \\
>14 \\
-\end{array}$ & $\begin{array}{l}>20 \\
>8 \\
>20 \\
-\end{array}$ & $\begin{array}{l}>20 \\
>8 \\
>15 \\
-\end{array}$ \\
\hline IV & $p_{T}^{\mathrm{tr}}$ & $>5$ & $>4$ & $>4$ & $>12$ & $>6$ & $>6$ \\
\hline V & $\begin{array}{l}m_{T}^{\mathrm{tr}} \\
m_{\ell_{1,2}, \mathrm{tr}}\end{array}$ & $\begin{array}{l}>10 \\
\notin[80,110]\end{array}$ & $\begin{array}{l}>10 \\
-\end{array}$ & $\begin{array}{l}>10 \\
-\end{array}$ & $\begin{array}{l}>10 \\
-\end{array}$ & $\begin{array}{l}>10 \\
<70\end{array}$ & $\begin{array}{l}>8 \\
<70\end{array}$ \\
\hline VI & anti $W$ & - & - & tight likelihood ${ }^{\mathrm{c}}$ & - & $\begin{array}{l}\text { tight likeli } \\
\text { hit in } 2 \text { in } \\
\text { very tight } \\
\sum_{0.05<\Delta \mathcal{R}}\end{array}$ & $i^{\mathrm{i}}{ }^{\mathrm{e}}$ \\
\hline VII & $\begin{array}{l}\notin_{T} \times p_{T}^{\text {tr }} \\
p_{T}^{\text {bal }}\end{array}$ & $\begin{array}{l}>200 \\
<4\end{array}$ & $\begin{array}{l}>300 \\
<4\end{array}$ & $\begin{array}{l}>220 \\
<4\end{array}$ & $\begin{array}{l}- \\
<4\end{array}$ & $\begin{array}{l}- \\
<2\end{array}$ & $\begin{array}{l}- \\
<2\end{array}$ \\
\hline
\end{tabular}

a $p_{T}^{\ell 1}$ and $p_{T}^{\ell 2}$ are electron and muon $p_{T}$, respectively.

b $\ell$ refers to the two identified leptons.

c For $p_{T}^{\text {tr }}<15 \mathrm{GeV}$.

d For $m_{T}^{\mu} \in[40,90] \mathrm{GeV}$.

e For $m_{T}^{e} \in[40,90] \mathrm{GeV}$.

The signature of $\tau$-type 1 ( $\tau$-type 2 ) consists of a single track with energy deposit in the hadronic (and the electromagnetic) calorimeter typically arising from $\pi^{ \pm}$-like $\left(\rho^{ \pm}\right.$-like) decays. Three-prong decays ( $\tau$-type 3 ) are not considered here, since the background contribution from jets in this channel does not allow one to improve the sensitivity to a signal. The separation of hadronic $\tau$ leptons and jets is based on a set of neural networks (NN), one for each $\tau$-type, exploiting the differences in longitudinal and transverse shower shapes as well as differences in the isolation in the calorimeter and the tracker [13]. $Z \rightarrow \tau \tau$ MC events are used as the signal training sample for the neural networks, while multijet events from data serve as the background training sample. In order to ensure high efficiency for low $\tau$ lepton transverse momenta, the selection on the NN output varies depending on the transverse momenta of the $\tau$ candidates to keep a constant efficiency of $60 \%$. At a small rate, muons can be misidentified as one-prong hadronic $\tau$ lepton decays, and thus $\tau$ candidates to which a muon can be matched are rejected.

Jets are reconstructed with an iterative midpoint cone algorithm [14] with cone radius of 0.5 and must be within $|\eta|<2.5$. The $\mathbb{E}_{T}$ is calculated from the vector sum of the transverse components of the energy deposited in the calorimeter cells and is corrected for electron, $\tau$ and jet energy calibrations as well as the transverse momentum of muons.

In the following, four different channels are defined, distinguished by the lepton content of the final state. For the di-electron plus lepton channel (ee $\ell$ ) two identified electrons are required using the electron identification criteria described above. In the dimuon plus lepton channel $(\mu \mu \ell)$, one tight and one loose muon are required, while the selection in the electron, muon plus lepton channel $(e \mu \ell)$ starts from one electron and one tight muon. Finally, the muon, $\tau$ lepton plus lepton channel $(\mu \tau)$ requires one tight muon and one hadronically decaying $\tau$ lepton in the final state. In all cases, unless explicitly specified otherwise, the third lepton is reconstructed as an isolated track without using the standard lepton identification criteria.
For each of the ee $\ell, \mu \mu \ell$ and $e \mu \ell$ channels, one "low- $p_{T}$ " and one "high- $p_{T}$ " selection is designed to exploit the different kinematic properties for various parameter points in the $m_{0}-m_{1 / 2}$ plane. The $\mu \tau$ channel is separated into two distinct selections based on the properties of the third object. One selection requires only an isolated track as third object, as in the other three analyses ( $\mu \tau \ell$ selection). For the second selection, a fully reconstructed hadronic $\tau$ lepton is required ( $\mu \tau \tau$ selection). Both $\mu \tau$ selections are identical over the whole $m_{0}-m_{1 / 2}$ plane.

Each selection requires two identified leptons stemming from the primary vertex with minimum transverse momenta of $p_{T}^{\ell 1}=$ $12 \mathrm{GeV}$ and $p_{T}^{\ell 2}=8 \mathrm{GeV}$. Due to higher thresholds in the single muon triggers used for the $\mu \tau$ channel, the $p_{T}$ cut on the muon is tightened to $15 \mathrm{GeV}$ for this channel. If more than two leptons are identified that satisfy the $p_{T}$ criteria, the two leptons with the highest $p_{T}$ are considered. In case of the $e \mu \ell$ analysis, events are removed if two electrons or muons with an invariant mass compatible with that of the $Z$ boson mass are found. This is called the preselection. To further reduce the background, differences in the kinematics and event topology compared to signal are exploited. All selection criteria are summarized in Tables 1 and 2 .

The dominant background from Drell-Yan and $Z$ boson production in the $\mu \mu \ell$ and eel channels as well as multijet background can be reduced by selecting on the invariant mass $m_{\ell_{1} \ell_{2}}$ of the identified di-lepton system and the opening angle $\Delta \phi_{\ell_{1} \ell_{2}}$ of the same two leptons in the transverse plane. As shown in Fig. 1, a major fraction of the di-lepton events from $Z$ boson decays can be rejected by requiring the invariant mass $m_{\ell_{1} \ell_{2}}$ to be below the $Z$ resonance. A substantial fraction of the Drell-Yan events as well as the major part of events from multijet production are back-to-back in the transverse plane and can be rejected by removing events with large opening angle $\Delta \phi_{\ell_{1} \ell_{2}}$.

Another striking feature of the signal is the presence of large $\mathbb{E}_{T}$ due to the escaping neutralinos and neutrinos in the final state. Thus selecting events with large $\not_{T}$ is expected to further enhance the signal, which is illustrated in Fig. 2. However, backgrounds 
Table 2

Criteria for the $\mu \tau \ell$ and $\mu \tau \tau$ selections (all energies, masses and momenta in $\mathrm{GeV}$, angles in radians), see text for further details.

\begin{tabular}{|c|c|c|c|c|}
\hline & Selection & $\mu \tau \ell$ & & $\mu \tau \tau$ \\
\hline I & $p_{T}^{\ell 1}, p_{T}^{\ell 2}$ & & $>15,>8^{a}$ & \\
\hline II & $\Delta \phi_{\ell_{1} \ell_{2}}$ & & $<2.9$ & \\
\hline . & $\begin{array}{l}\not k_{T} \\
\operatorname{Sig}\left(\not \not_{T}\right) \\
m_{T}^{\mu} \\
\text { jet-veto } H_{T}\end{array}$ & & $\begin{array}{l}>20 \\
>8 \\
>20 \\
<80\end{array}$ & \\
\hline IV & $p_{T}^{\mathrm{tr}}$ & $>3$ & $p_{T}^{\tau_{2}}$ & $>4$ \\
\hline V & $\begin{array}{l}\Delta \phi_{\mathrm{tr}, \mathrm{E}_{T}} \\
m_{\ell_{1,2}, \mathrm{tr}}\end{array}$ & $\begin{array}{l}>0.5 \\
<60\end{array}$ & $\Delta \phi_{\tau_{2}, k_{T}}$ & $\begin{array}{l}>0.5 \\
<60\end{array}$ \\
\hline VI & anti $W$ & likelihood & $N N_{\tau_{1}} \times N N_{\tau_{2}}$ & $\begin{array}{l}\text { likelihood } \\
>0.7\end{array}$ \\
\hline VII & $\not_{T} \times p_{T}^{\mathrm{tr}}$ & $>300$ & $p_{T}^{\text {bal }}$ & $<3.5$ \\
\hline
\end{tabular}

a $p_{T}^{\ell 1}$ and $p_{T}^{\ell 2}$ are muon and $\tau$ lepton $p_{T}$, respectively.

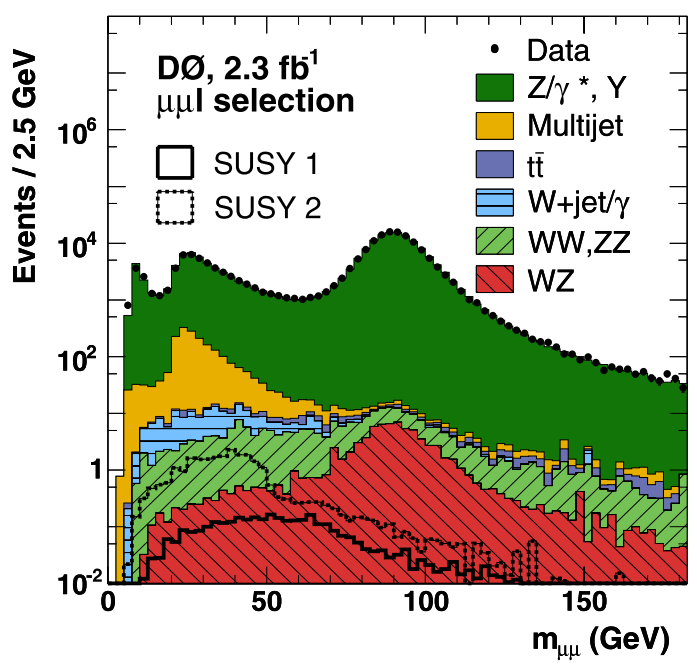

Fig. 1. Invariant mass $m_{\mu \mu}$ ( $\mu \mu \ell$ channel) for data (points), SM backgrounds (shaded histograms), and SUSY signal (open histograms) after cut I (see Table 1) for the low- $p_{T}$ selection.

without true $\mathbb{E}_{T}$ can potentially satisfy this selection criterion, because of mismeasurements of the objects in the event or by failing to reconstruct them. If $\mathbb{E}_{T}$ is caused by mismeasurement of an object, the direction of the $\not_{T}$ tends to be aligned with this object. We use the expected jet energy resolution as an estimate of potential mismeasurements resulting in missing transverse energy. To evaluate how much a given jet might have contributed to $\ddot{E}_{T}$, we project the jet transverse momentum onto the direction of $\not_{T}$, and calculate the resolution $\sigma\left(p_{T}^{j} \| \mathbb{E}_{T}\right)=\sigma\left(p_{T}^{j}\right) \cdot \cos \phi\left(j, \mathbb{E}_{T}\right)$ of that momentum component. For events with at least one jet, $\operatorname{Sig}\left(\mathbb{E}_{T}\right)$ is defined as

$\operatorname{Sig}\left(\mathbb{E}_{T}\right)=\frac{\mathbb{E}_{T}}{\sqrt{\sum_{\mathrm{jets}} \sigma^{2}\left(p_{T}^{j} \| \mathbb{E}_{T}\right)}}$.

As a result, $\operatorname{Sig}\left(\mathbb{E}_{T}\right)$ is expected to be small for events with poorly measured jets. Rejecting events with small minimal transverse mass $m_{T}^{\min }=\min \left(m_{T}^{\ell_{1}}, m_{T}^{\ell_{2}}\right)$, where $m_{T}^{\ell}=\left\{2 p_{T}^{\ell} \notin_{T}[1-\right.$ $\left.\left.\cos \Delta \phi\left(\ell, \mathscr{E}_{T}^{\dagger}\right)\right]\right\}^{1 / 2}$, removes events with mismeasured leptons as illustrated in Fig. 3. Other events with large jet activity, in particular $t \bar{t}$ production, can be removed with a cut on $H_{T}$, the scalar sum of the $p_{T}$ of all jets with $p_{T}>15 \mathrm{GeV}$.

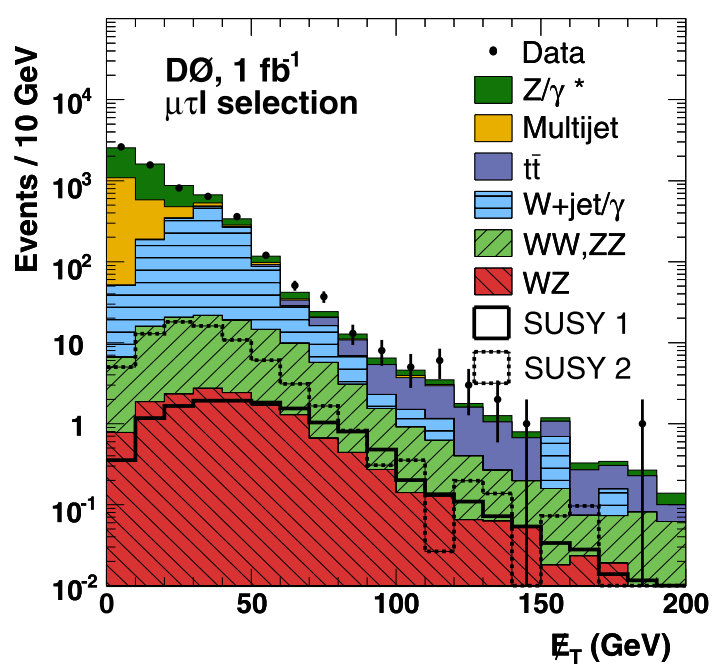

Fig. 2. Missing transverse energy $\not_{T}$ ( $\mu \tau \ell$ selection) for data (points), SM backgrounds (shaded histograms), and SUSY signal (open histograms) after cut I (see Table 2).

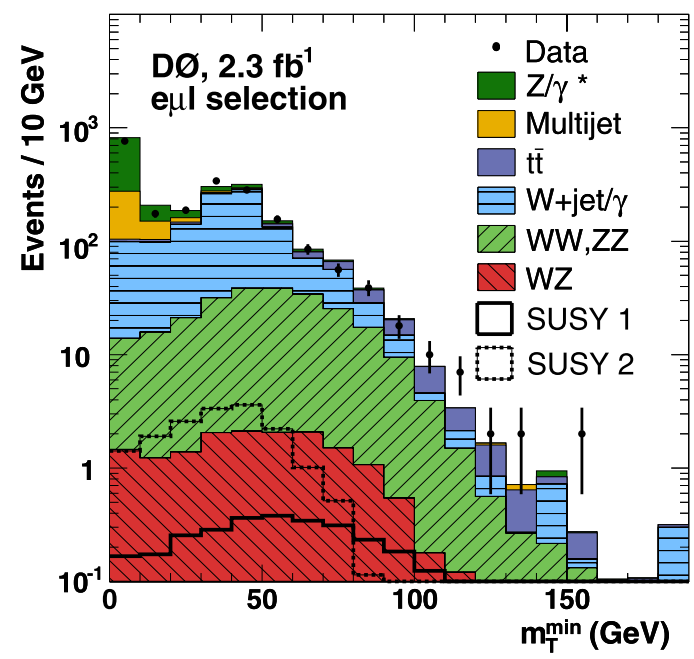

Fig. 3. Minimum transverse mass $m_{T}^{\min }$ ( $e \mu \ell$ channel) for data (points), SM backgrounds (shaded histograms), and SUSY signal (open histograms) before applying the cut on $m_{T}^{\min }$ (see Table 1 ) for the low- $p_{T}$ selection.

Unlike most SM backgrounds, signal events contain three charged leptons. This can be exploited to remove most of the remaining background, which is dominated by $W+$ jet production at this stage of the selection. The eel, $\mu \mu \ell, e \mu \ell$, and $\mu \tau \ell$ selections only require an additional track that must be isolated in both the tracking system and the calorimeter as indication of this third lepton. Dropping the lepton identification criteria in this case increases the signal efficiency and includes all three lepton flavors in the selection. The distribution of the transverse momentum of this additional track is presented in Fig. 4 after $\mathbb{E}_{T}$, $\operatorname{Sig}\left(\not_{T}\right)$ and $m_{T}^{\text {min }}$ cuts are applied. Selection of tracks with high transverse momentum clearly enhances signal over background. For the $\mu \tau \tau$ channel, a well-identified second $\tau$ lepton is required instead of the track. Since the $\tau$ lepton selection imposes different criteria than the track selection, some signal loss due to the third track criterion can be regained using this selection. In particular at high $\tan \beta$, this selection is favored, since most of the leptons in the final state are expected to be $\tau$ leptons. Fig. 5 shows the distribution of the transverse momentum for the second $\tau$ lepton candidate. 


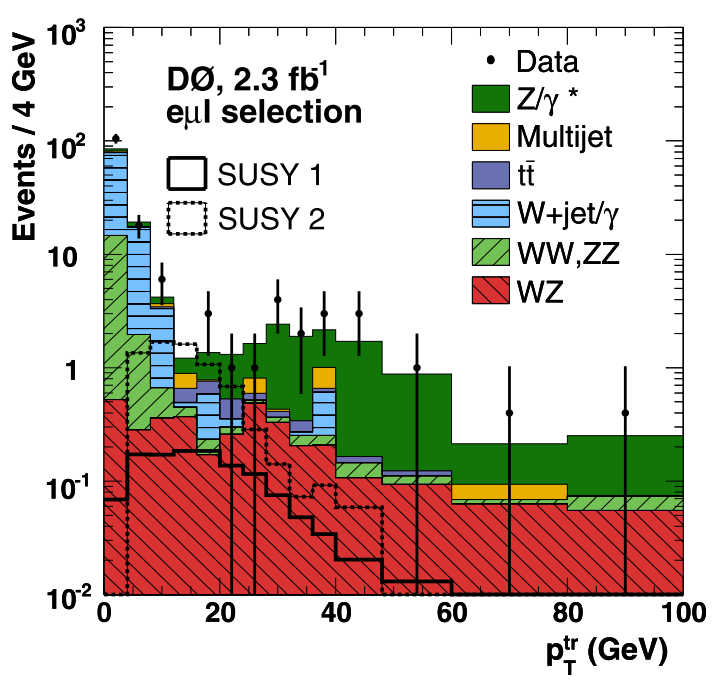

Fig. 4. Transverse momentum of the track ( $e \mu \ell$ channel) for data (points), SM backgrounds (shaded histograms), and SUSY signal (open histograms) after all $\notin_{T}$ related cuts are applied (cut III, see Table 1) for the low- $p_{T}$ selection.

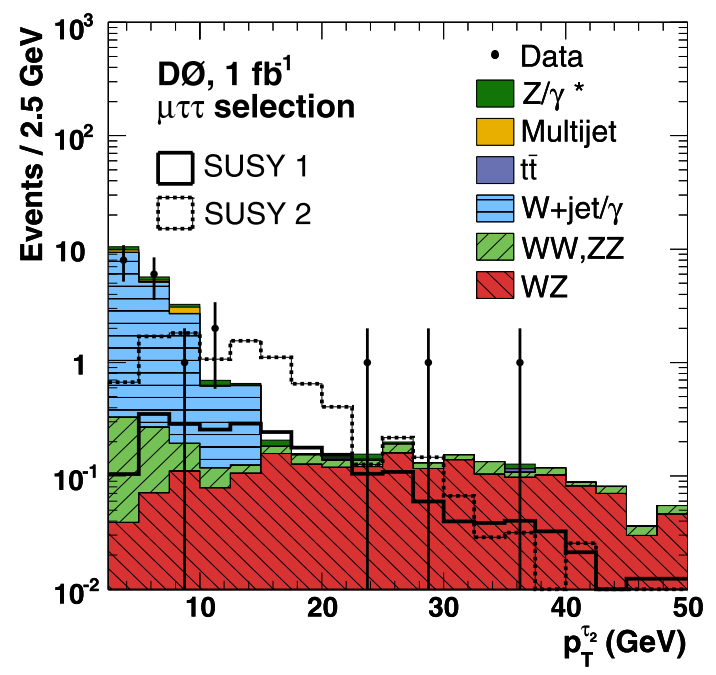

Fig. 5. Transverse momentum of the second $\tau$ lepton candidate ( $\mu \tau \tau$ selection) for data (points), SM backgrounds (shaded histograms), and SUSY signal (open histograms) after cut III (see Table 2).

After the third object selection, the remaining background consists mainly of $W$ and $Z$ boson as well as di-boson production. These backgrounds are addressed in the following. The remaining $Z$ boson background mainly consists of events where one of the leptons from the $Z$ boson decay is not reconstructed in the calorimeter or muon system, but instead a jet or photon from initial or final state radiation is misidentified as one of the two initially selected leptons. However, the missed lepton from the $Z$ boson decay is then selected as the third track. This unique feature provides two handles to reject this background. Due to the non-reconstruction of one of the leptons, the $\mathbb{k}_{T}$ tends to point into the direction of the track. Thus the transverse mass calculated from the track and $\mathbb{E}_{T}$ should be small due to the small opening angle $\Delta \phi_{\mathrm{tr}, \mathbb{E}_{T}}$. In addition, the invariant mass of the track and one of the leptons, $m_{\ell_{1,2} \text {,tr }}$, is expected to be consistent with the $Z$ boson mass. The same is true for $W Z$ production, where again one of the leptons from the $Z$ decay is reconstructed in the tracking system.

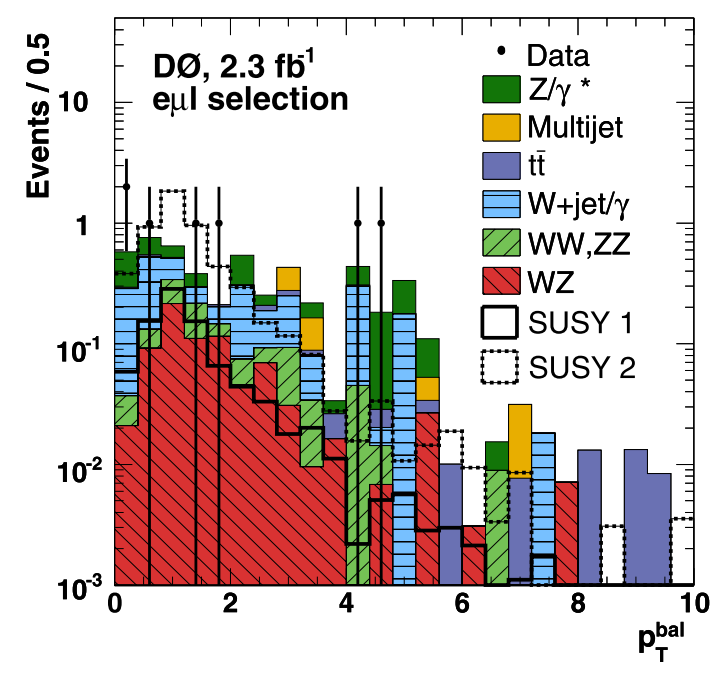

Fig. 6. Transverse momentum balance $p_{T}^{\text {bal }}$ ( $e \mu \ell$ channel) for data (points), SM backgrounds (shaded histograms), and SUSY signal (open histograms) after cut V (see Table 1) for the low- $p_{T}$ selection.

For $W$ boson production, only one real lepton is expected from the decay of the $W$ boson, the second lepton is mimicked by a jet or a photon. In the case of jets, the identification criteria for that lepton tend to be of worse quality, while in case of photon conversions, no hits in the innermost layers of the tracking detector are expected for the track corresponding to the converted photon. Thus, requiring high quality leptons (tight likelihood for electrons and very tight track isolation for muons) or hits in the first two layers of the tracking system is expected to reduce $W+$ jet $/ \gamma$ background. To keep signal efficiencies high, these requirements are only used if the event properties and kinematics are similar to expectations from $W$ boson production (see Table 1 ). In case of the $\mu \tau \ell$ selection, a dedicated likelihood discriminant is developed to remove the background from $W$ boson production. This likelihood uses the transverse masses calculated for all of the three leptons as well as products of $\mathbb{E}_{T}$ and lepton transverse momenta. In case of the $\mu \tau \tau$ selection, the product of the two NN outputs for $\tau$ lepton identification is used to remove events containing misidentified $\tau$ candidates.

Finally, the different event kinematics for signal and background are exploited to obtain better signal sensitivity. Since background is expected to have low transverse momentum for the third track or small $\ddot{E}_{T}$, a cut on the product of track $p_{T}$ and $\ddot{k}_{T}$ effectively rejects any remaining background contributions. In addition, the vectorial sum of the lepton transverse momenta and $\mathbb{E}_{T}$ should equal the transverse momentum of the third track in case of signal events. Thus the $p_{T}$ balance

$p_{T}^{\text {bal }}=\frac{\left|\vec{p}_{T}^{\ell_{1}}+\vec{p}_{T}^{\ell_{2}}+\vec{E}_{T}\right|}{p_{T}^{\text {tr }}}$

is expected to peak at 1 for a signal, while for background a broad distribution is expected. This is illustrated in Fig. 6 .

After all selection criteria are applied, the expected background is dominated by irreducible background from $W Z$ production, as is evident from the marginal distribution of the di-electron invariant mass in the eel selection shown in Fig. 7. A detailed comparison of background expectation and events observed in data together with efficiency expectations from a typical SUSY signal are shown in Tables 3 and 4 for the low- $p_{T}$ and high- $p_{T}$ selection, respectively, while Table 5 presents the results for the $\mu \tau$ selections. In general, good agreement between data and expectation from SM processes is observed. Combining all low- $p_{T}$ and $\mu \tau$ selections, a 
Table 3

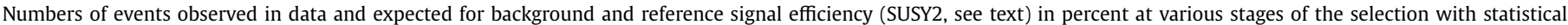
uncertainties for the low- $p_{T}$ selection. Each row corresponds to a group of cuts, as detailed in Table 1 .

\begin{tabular}{|c|c|c|c|c|c|c|c|c|c|}
\hline \multirow[t]{2}{*}{ Selection } & \multicolumn{3}{|l|}{$\mu \mu \ell$} & \multicolumn{3}{|l|}{ eel } & \multicolumn{3}{|l|}{$e \mu \ell$} \\
\hline & Data & Backgrd. & Eff. (\%) & Data & Backgrd. & Eff. (\%) & Data & Backgrd. & Eff. (\%) \\
\hline I & 194006 & $195557 \pm 177$ & $19.9 \pm 0.3$ & 235474 & $232736 \pm 202$ & $15.5 \pm 0.2$ & 16630 & $16884 \pm 75$ & $10.5 \pm 0.1$ \\
\hline II & 22766 & $26067 \pm 88$ & $14.6 \pm 0.2$ & 31365 & $27184 \pm 64$ & $11.0 \pm 0.2$ & & & \\
\hline III & 178 & $181 \pm 6.4$ & $8.8 \pm 0.1$ & 515 & $512 \pm 12$ & $6.8 \pm 0.2$ & 1191 & $1177 \pm 20$ & $5.8 \pm 0.1$ \\
\hline IV & 7 & $2.9 \pm 0.7$ & $3.4 \pm 0.1$ & 16 & $9.3 \pm 2.0$ & $3.0 \pm 0.1$ & 22 & $18.0 \pm 1.2$ & $2.4 \pm 0.1$ \\
\hline V & 4 & $2.2 \pm 0.5$ & $3.0 \pm 0.1$ & 9 & $5.9 \pm 1.7$ & $2.5 \pm 0.1$ & 3 & $3.5 \pm 0.5$ & $2.0 \pm 0.1$ \\
\hline VI & & & & 6 & $3.1 \pm 0.4$ & $2.2 \pm 0.1$ & 2 & $1.6 \pm 0.4$ & $1.5 \pm 0.1$ \\
\hline VII & 4 & $1.2 \pm 0.2$ & $2.8 \pm 0.1$ & 2 & $1.8 \pm 0.2$ & $2.1 \pm 0.1$ & 2 & $0.8 \pm 0.2$ & $1.3 \pm 0.1$ \\
\hline
\end{tabular}

Table 4

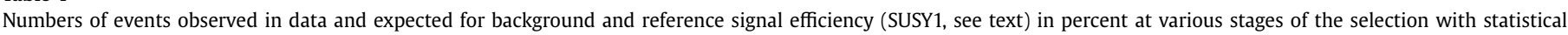
uncertainties for the high- $p_{T}$ selection. Each row corresponds to a group of cuts, as detailed in Table 1 .

\begin{tabular}{|c|c|c|c|c|c|c|c|c|c|}
\hline \multirow[t]{2}{*}{ Selection } & \multicolumn{3}{|l|}{$\mu \mu \ell$} & \multicolumn{3}{|l|}{ 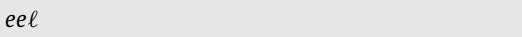 } & \multicolumn{3}{|l|}{$e \mu \ell$} \\
\hline & Data & Backgrd. & Eff. (\%) & Data & Backgrd. & Eff. (\%) & Data & Backgrd. & Eff. (\%) \\
\hline I & 140417 & $141781 \pm 120$ & $19.6 \pm 0.2$ & 171001 & $170197 \pm 175$ & $18.1 \pm 0.2$ & 4617 & $4709 \pm 23$ & $11.5 \pm 0.2$ \\
\hline II & 10349 & $10645 \pm 51$ & $15.3 \pm 0.2$ & 8273 & $7937 \pm 39$ & $12.8 \pm 0.1$ & & & \\
\hline III & 173 & $176 \pm 5.7$ & $11.4 \pm 0.2$ & 244 & $264 \pm 10$ & $10.8 \pm 0.1$ & 727 & $738 \pm 11$ & $8.9 \pm 0.1$ \\
\hline IV & 7 & $3.8 \pm 0.5$ & $5.9 \pm 0.1$ & 0 & $1.5 \pm 0.3$ & $4.0 \pm 0.1$ & 11 & $12.7 \pm 0.9$ & $4.1 \pm 0.1$ \\
\hline V & 4 & $2.9 \pm 0.4$ & $5.5 \pm 0.1$ & 0 & $1.1 \pm 0.3$ & $3.6 \pm 0.1$ & 2 & $2.8 \pm 0.5$ & $2.9 \pm 0.1$ \\
\hline VI & & & & & & & 0 & $1.0 \pm 0.2$ & $2.4 \pm 0.1$ \\
\hline VII & 4 & $2.0 \pm 0.3$ & $5.0 \pm 0.1$ & 0 & $0.8 \pm 0.1$ & $3.6 \pm 0.1$ & 0 & $0.5 \pm 0.1$ & $2.1 \pm 0.1$ \\
\hline
\end{tabular}

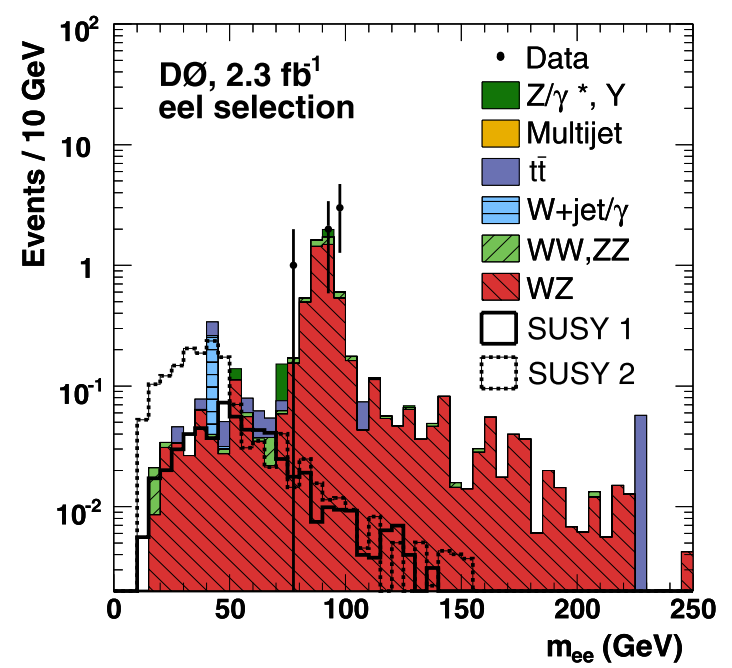

Fig. 7. Distribution of the invariant mass $m_{e e}$ (ee $\ell$ channel) for data (points), SM backgrounds (shaded histograms), and SUSY signal (open histograms) with all cuts applied except the $m_{e e}$ requirement for the low- $p_{T}$ selection.

background of $5.4 \pm 0.4$ (stat) \pm 0.4 (syst) events from SM processes is expected with 9 events observed in the data. The probability to observe 9 or more events in the data given the expected background is $10 \%$. The expectation for the reference signal point SUSY2 is $9.3 \pm 0.3$ (stat) \pm 0.8 (syst) events. The high- $p_{T}$ selection yields $3.3 \pm 0.3$ (stat) \pm 0.3 (syst) events from SM processes, while 4 events are observed in data. The expected reference signal for parameter point SUSY1 is $0.9 \pm 0.1$ (stat) \pm 0.1 (syst) events.

The estimate of the expected numbers of signal and background events depends on various measurements with associated systematic uncertainties: integrated luminosity (6\%) [15], trigger efficiency, lepton identification and reconstruction efficiencies (4\%), jet and $\tau$ energy calibration in signal (2-6\%) and background events (2-9\%), PDF uncertainties (3-4\%), and modeling of the multijet background (2-30\%). All uncertainties, except the last one, are correlated among the different channels.
Table 5

Numbers of events observed in data and expected for background and reference signal efficiency (SUSY2 for the $\mu \tau \ell$ selection and SUSY1 for the $\mu \tau \tau$ selection, see text) in percent at various stages of the selection with statistical uncertainties for the $\mu \tau$ selections. Each row corresponds to a group of cuts, as detailed in Table 2.

\begin{tabular}{|c|c|c|c|c|c|c|}
\hline \multirow[t]{2}{*}{ Selection } & \multicolumn{3}{|l|}{$\mu \tau \ell$} & \multicolumn{3}{|l|}{$\mu \tau \tau$} \\
\hline & Data & Backgrd. & Eff. (\%) & Data & Backgrd. & Eff. (\%) \\
\hline I & 6251 & $6238 \pm 30$ & $8.1 \pm 0.2$ & 6251 & $6238 \pm 30$ & $12.4 \pm 0.2$ \\
\hline II & 3473 & $3416 \pm 17$ & $6.9 \pm 0.2$ & 3473 & $3416 \pm 17$ & $10.8 \pm 0.2$ \\
\hline III & 1180 & $1154 \pm 14$ & $4.5 \pm 0.1$ & 1180 & $1154 \pm 14$ & $8.7 \pm 0.1$ \\
\hline IV & 103 & $110.0 \pm 5.1$ & $2.9 \pm 0.1$ & 20 & $22.6 \pm 2.6$ & $2.2 \pm 0.1$ \\
\hline V & 67 & $52.8 \pm 4.1$ & $2.1 \pm 0.1$ & 7 & $8.0 \pm 1.5$ & $1.7 \pm 0.1$ \\
\hline VI & 4 & $2.9 \pm 0.4$ & $1.5 \pm 0.1$ & 3 & $1.9 \pm 0.5$ & $1.4 \pm 0.1$ \\
\hline VII & 0 & $0.8 \pm 0.1$ & $1.2 \pm 0.1$ & 1 & $0.8 \pm 0.2$ & $1.3 \pm 0.1$ \\
\hline
\end{tabular}

No evidence for a signal is observed. The search results can be translated into upper limits on the product of cross section and branching fraction into three charged leptons, $\sigma \mathrm{BR}(3 \ell)$. Limits are based on the combination of all low- and high- $p_{T}$ selections. Events appearing in multiple analyses are uniquely assigned to the channel with the best signal to background ratio. Correlated systematic uncertainties are taken into account.

To calculate the limits, the mass relations between the particles involved in the decay chain of chargino and neutralino have to be known. The mSUGRA model is used to calculate the mass differences between $\tilde{\chi}_{1}^{ \pm}, \tilde{\chi}_{2}^{0}$, and $\tilde{\chi}_{1}^{0}$, which approximately corresponds to the assumption $m_{\tilde{\chi}_{1}^{ \pm}} \approx m_{\tilde{\chi}_{2}^{0}} \approx 2 m_{\tilde{\chi}_{1}^{0}}$. For slepton and sneutrino masses, several scenarios are taken into account.

Fig. 8 shows the limit on $\sigma \mathrm{BR}(3 \ell)$ as a function of chargino mass assuming that sleptons and sneutrinos are heavier than the lightest chargino and the second-lightest neutralino, and assuming that slepton mixing can be neglected. In this case, both $\tilde{\chi}_{1}^{ \pm}$ and $\tilde{\chi}_{2}^{0}$ decay via three-body decays, and branching fractions do not depend on the lepton flavor. The limit is compared with the NLO cross section [16] multiplied by branching fractions calculated in the limit of infinitely heavy sleptons ("large- $m_{0}$ " scenario) and for slepton masses just above the mass of the $\tilde{\chi}_{2}^{0}$, in which case the leptonic branching fraction for three-body decays is maximized ("31-max" scenario). To calculate the cross section and branching fractions for the 31-max scenario, $m_{0}$ has been adjusted such 


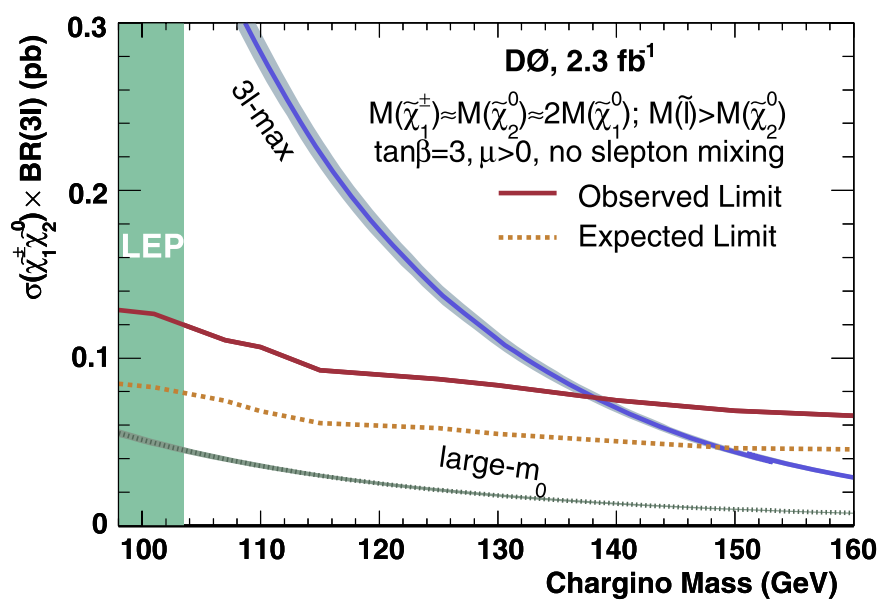

Fig. 8. Upper limit at the $95 \%$ C.L. on $\sigma \mathrm{BR}(3 \ell)$ as a function of $\tilde{\chi}_{1}^{ \pm}$mass, in comparison with the expectation for two SUSY scenarios (see text). PDF and renormalization/factorization scale uncertainties on the predicted cross section are shown as shaded bands.

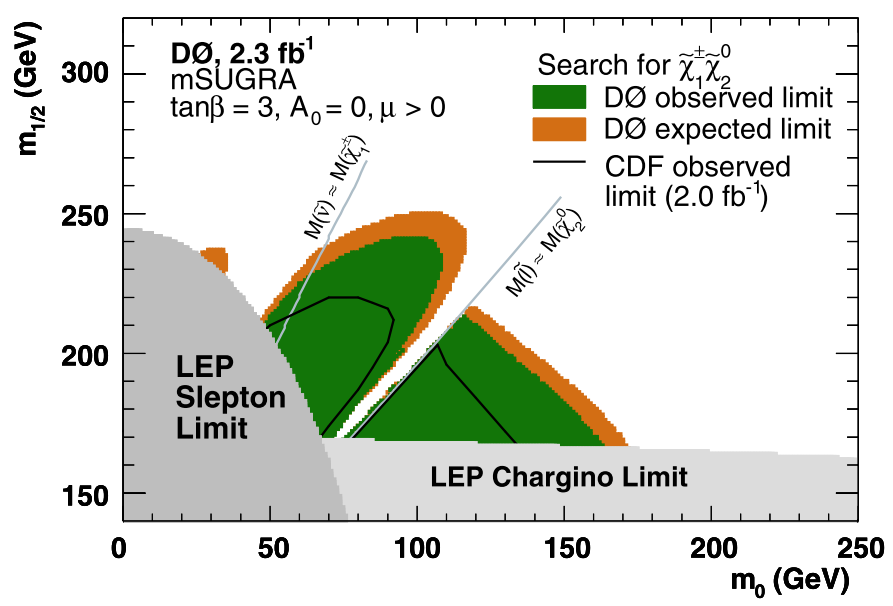

Fig. 9. Region in the $m_{0}-m_{1 / 2}$ plane excluded by the combination of the $\mathrm{D} \emptyset$ analyses (green), by LEP searches for charginos (light grey) and sleptons (dark grey) [2] and CDF (black line) [4]. The assumed mSUGRA parameters are $\tan \beta=3, A_{0}=0$ and $\mu>0$.

that the mass difference between right-handed sleptons and the second-lightest neutralino is $1 \mathrm{GeV}$. In this case, an observed (expected) lower limit at the 95\% C.L. on the chargino mass is set at $138 \mathrm{GeV}(148 \mathrm{GeV})$.

Alternatively, the results can be interpreted within mSUGRA. To obtain the efficiency for any given point in the $m_{0}-m_{1 / 2}$ plane, selection efficiencies are first determined separately for three-body decays of chargino and neutralino as well as two-body decays via sleptons and sneutrinos. The variation of these efficiencies throughout the plane can then be parametrized for each selection as a function of the chargino, slepton and sneutrino masses. Using the mSUGRA prediction of branching fractions and masses $[6,11,17]$, the parametrized efficiencies are used to calculate the total efficiency for each point in the $m_{0}-m_{1 / 2}$ plane. Fig. 9 shows the region excluded in the $m_{0}-m_{1 / 2}$ plane for $\tan \beta=3, A_{0}=0$ and $\mu>0$ in comparison with the limits from chargino and slepton searches at LEP [2] and CDF [4]. The difference in sensitivity between the CDF result and the one presented here can mostly be attributed to differences in lepton acceptance of the two analyses. The shape of the excluded region is driven by the relation of gaugino and slepton masses throughout the plane, which affects the branching fraction into three charged leptons as well as the

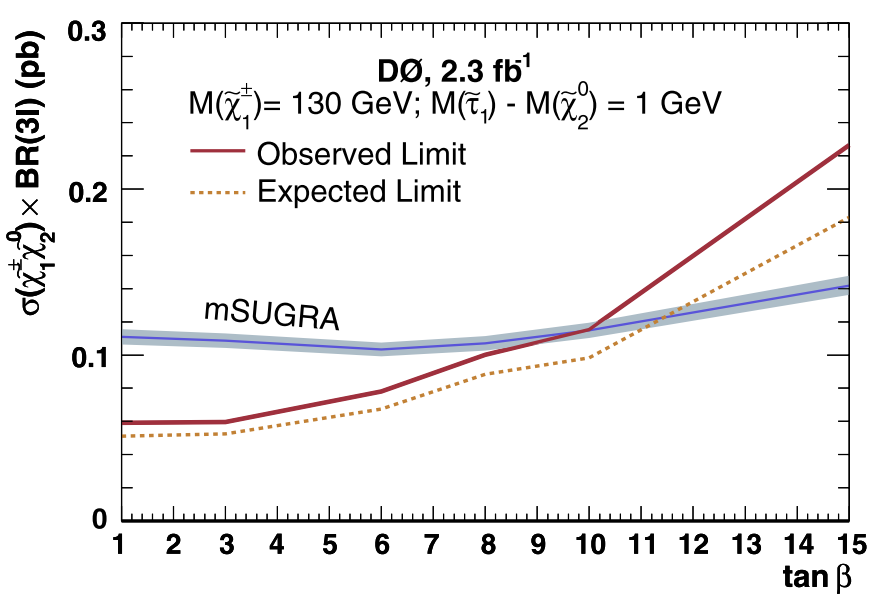

Fig. 10. Upper limit at the 95\% C.L. on $\sigma \mathrm{BR}(3 \ell)$ as a function of $\tan \beta$ in comparison with the prediction for a chargino mass of $130 \mathrm{GeV}$ and $m_{\tilde{\tau}}-m_{\tilde{\chi}_{2}^{0}}=1 \mathrm{GeV}$.

efficiencies of the selections. For slepton masses just below the $\tilde{\chi}_{2}^{0}$ mass, one of the leptons from the $\tilde{\chi}_{2}^{0}$ decay has very small momentum, rendering the trilepton selections inefficient. For sneutrinos lighter than the $\tilde{\chi}_{1}^{ \pm}$and $\tilde{\chi}_{2}^{0}$, two-body decays into sneutrinos open up, leading to a smaller branching fraction into three charged leptons as well as a reduced selection efficiency due to the small mass difference between sneutrino and chargino. For the intermediate region at $m_{1 / 2} \approx 245 \mathrm{GeV}$, chargino decays via $W$ bosons compete with decays via sleptons, leading to a reduction in leptonic branching fraction with increasing $m_{1 / 2}$ both below and above the threshold for production of a real $W$ boson.

The excluded region in the $m_{0}-m_{1 / 2}$ plane depends on the choice of $\tan \beta$, as the branching fraction into $\tau$ leptons increases as a function of $\tan \beta$. Fig. 10 shows the limit on $\sigma \mathrm{BR}(3 \ell)$ as a function of $\tan \beta$ for a chargino mass of $130 \mathrm{GeV}$ and fixing $m_{0}$ such that the lightest stau $\left(\tilde{\tau}_{1}\right)$ is heavier than the $\tilde{\chi}_{2}^{0}$ by $1 \mathrm{GeV}$. The latter choice results in three-body decays with maximal leptonic branching fraction. The leptonic branching fraction into three $\tau$ leptons increases as a function of $\tan \beta$, reaching values above $50 \%$ for $\tan \beta>15$. Because all selections have been designed to be efficient for $\tau$ leptons, the limit remains stable within a factor of two for $\tan \beta \lesssim 10$, allowing one to exclude charginos with a mass of $130 \mathrm{GeV}$ up to $\tan \beta$ of 9.6 .

To summarize, a data set collected with the DØ detector corresponding to an integrated luminosity of $2.3 \mathrm{fb}^{-1}$ has been analyzed in search of the associated production of charginos and neutralinos in final states with three charged leptons and $\not_{T}$. No evidence for a signal is observed, and upper limits on the product of production cross section and leptonic branching fraction have been set. Within the reference model of mSUGRA with $\tan \beta=3$, $A_{0}=0$, and $\mu>0$, this result translates into excluded regions in the $m_{0}-m_{1 / 2}$ plane that significantly extend beyond all existing limits from direct searches for supersymmetric particles.

\section{Acknowledgements}

We thank the staffs at Fermilab and collaborating institutions, and acknowledge support from the DOE and NSF (USA); CEA and CNRS/IN2P3 (France); FASI, Rosatom and RFBR (Russia); CNPq, FAPERJ, FAPESP and FUNDUNESP (Brazil); DAE and DST (India); Colciencias (Colombia); CONACyT (Mexico); KRF and KOSEF (Korea); CONICET and UBACyT (Argentina); FOM (The Netherlands); STFC (United Kingdom); MSMT and GACR (Czech Republic); CRC Program, CFI, NSERC and WestGrid Project (Canada); BMBF and DFG (Germany); SFI (Ireland); The Swedish Research Council (Swe- 
den); CAS and CNSF (China); and the Alexander von Humboldt Foundation (Germany).

\section{References}

[1] H.E. Haber, G.L. Kane, Phys. Rep. 117 (1985) 75.

[2] LEPSUSYWG, ALEPH, DELPHI, L3, OPAL Collaborations, notes LEPSUSYWG/0103.1 and 04-01.1, http://lepsusy.web.cern.ch/lepsusy/Welcome.html.

[3] V.M. Abazov, et al., DØ Collaboration, Phys. Rev. Lett. 95 (2005) 151805.

[4] T. Aaltonen, et al., CDF Collaboration, Phys. Rev. Lett. 101 (2008) 251801.

[5] V.M. Abazov, et al., Dø Collaboration, Nucl. Instrum. Methods Phys. Res. A 565 (2006) 463.

[6] T. Sjöstrand, et al., Comput. Phys. Commun. 135 (2001) 238.
[7] M.L. Mangano, et al., JHEP 0307 (2003) 001.

[8] R. Brun, F. Carminati, CERN Program Library Long Writeup W5013, 1993 (unpublished).

[9] J. Pumplin, et al., JHEP 0207 (2002) 012;

D. Stump, et al., JHEP 0310 (2003) 046.

[10] H.P. Nilles, Phys. Rep. 110 (1984) 1.

[11] B.C. Allanach, Comput. Phys. Commun. 143 (2002) 305

[12] T. Junk, Nucl. Instrum. Methods A 434 (1999) 435.

[13] V.M. Abazov, et al., Dø Collaboration, Phys. Lett. B 670 (2009) 292.

[14] G.C. Blazey, et al., arXiv:hep-ex/0005012.

[15] T. Andeen, et al., FERMILAB-TM-2365 (2007).

[16] W. Beenakker, et al., Phys. Rev. Lett. 83 (1999) 3780;

W. Beenakker, et al., Phys. Rev. Lett. 100 (2008) 029901, Erratum.

[17] M. Mühlleitner, A. Djouadi, Y. Mambrini, Comput. Phys. Commun. 168 (2005) 46. 